\title{
Opposite Direction for Seasonal Variation of Aflatoxin M1 in Bulk Milk and Aflatoxin B1 in Rations: Results From a Prospective Study in Selected Dairy Farms of Qazvin Province, Iran
}

Ravel Gholampour-Aghdami

Shiraz University School of Veterinary Medicine

Mehdi Mohebbi-Fani

Shiraz University School of Veterinary Medicine

Arash Omidi ( $\square$ aomidi@shirazu.ac.ir)

Shiraz University School of Veterinary Medicine https://orcid.org/0000-0001-9323-2721

Aria Rasooli

Shiraz University School of Veterinary Medicine

Maryam Maryam Ansari-Lari

Shiraz University School of Veterinary Medicine

Research Article

Keywords: Aflatoxin, Bulk milk, Dairy cows, Ration

Posted Date: December 8th, 2021

DOI: https://doi.org/10.21203/rs.3.rs-1120630/v1

License: (c) (i) This work is licensed under a Creative Commons Attribution 4.0 International License. Read Full License 


\section{Abstract}

The presence of aflatoxin $\mathrm{M}_{1}\left(\mathrm{AFM}_{1}\right)$ in $24 \mathrm{~h}$ bulk milk and aflatoxin $\mathrm{B}_{1}\left(\mathrm{AFB}_{1}\right)$ in concurrent total mixed rations (TMR) and feed ingredients were assessed in 12 large dairy operations. The bulk milk was sampled on days 1, 15 and 30 during winter and summer $(n=72)$. Total mixed rations ( $n=48)$ and feed ingredients $(n=230)$ were sampled two times with a 30-day interval. Aflatoxin was measured using direct competitive ELISA kits with detection limits of $1-81$ ngkg ${ }^{-1}$ for milk and 1.25-101.25 $\mathrm{ngkg}^{-1}$ for feeds. Aflatoxin $\mathrm{M}_{1}$ was identified in all milk samples (100\%), ranging from 2.03 to $>81 \mathrm{ngkg}^{-1}$, with a median of $70 \mathrm{ngkg}{ }^{-1}$ and averaging $61.25 \pm 28.91 \mathrm{ngkg}^{-1}$ in winter and $54.20 \pm 25.51 \mathrm{ngkg}^{-1}$ in summer $\left(\mathrm{P}=0.279\right.$ ). Contaminations $<81 \mathrm{ngkg}^{-1}$ (below the Iranian standard of 100 $\left.\mathrm{ngkg}^{-1}\right)$ were detected in $76 \%(\mathrm{n}=55 / 72)$ of samples. Contaminations $>81 \mathrm{ngkg}^{-1}$ were detected in $24 \%(\mathrm{n}=17 / 72)$ of samples and were more frequent in winter than in summer ( $42 \%$ vs. $6 \%)$. Sixty-nine percent of the winter milk samples $(n=25 / 36)$ had contaminations above the median $\left(70 \mathrm{ngkg}{ }^{-1}\right)$. A reverse result was detected in summer. The chance of contaminations above median was higher in winter than in summer $(\mathrm{OR}=5.33, \mathrm{P}=0.007)$. All TMR and ingredient samples had higher $A_{F B}$ contaminations in summer $(P<0.05)$. Six TMR samples had non-detectable $\left(<1.25 \mathrm{ngkg}^{-1}\right)$ values $(5$ in winter $)$ and 7 samples had levels $>101.25 \mathrm{ngkg}^{-1}$ (all in summer). The chance of TMR contamination above the median $\left(716 \mathrm{ngkg}^{-1}\right)$ was 5.57 times higher in summer than in winter $(P=0.002)$. Seventy percent of the TMR samples had contaminations above the median in summer. Elevated levels of $A F B_{1}$ of rations in summer

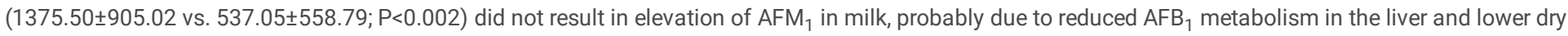
matter intakes caused by heat stress. The $\mathrm{AFB}_{1}$ content of grain mix succeeded by corn silage, wet beet pulp, dry beet pulp and alfalfa hay were correlated with TMR contamination. Ration $\mathrm{AFB}_{1}$ and milk $\mathrm{AFM}_{1}$ were not correlated. Based on the results, a great majority of milk produced in the studied farms could have $\mathrm{AFM}_{1}$ contaminations below the Iranian standard limit $\left(100 \mathrm{ngkg}^{-1}\right)$. Contaminations below $50 \mathrm{ngkg}^{-1}$ appear to be achievable and affordable. Intensifying the controlling measures in summer, when the feed contaminations are elevated, may reduce the overall milk contamination.

\section{Introduction}

Aflatoxin $\mathrm{M}_{1}\left(\mathrm{AFM}_{1}\right)$, a resistant immunosuppressive and carcinogenic toxin for humans, is secreted into milk after hepatic bio-transformation of dietary aflatoxin $\mathrm{B}_{1}\left(\mathrm{AFB}_{1}\right)$ (Gallo et al., 2015; Alvarado et al, 2017). Many types of feeds, including common feeds of dairy cows, may be contaminated to AFB (Alvarado et al., 2017; Bahrami et al., 2016; Kocasari et al., 2013; Sahin et al., 2013, 2016; Zheng et al., 2013). Widespread contamination of feedstuffs by aflatoxins necessitates some regular actions in dairy farms for hazard analysis and defining the critical control points. Aflatoxin regulatory limits vary between countries based on their specific considerations. The most proposed limits for $\mathrm{AFM}_{1}$ in raw milk are $50 \mathrm{ngkg}^{-1}$ (as low as reasonably achievable) and 500 $\mathrm{ngkg}^{-1}$ (regards to the carcinogenic effects of $\mathrm{AFM}_{1}$ ) (FAO/WHO, 2002; 2001). It has been stated in some FAO/WHO reports (2001; 2002) that 500 ngkg-1 $\mathrm{AFM}_{1}$ may be high because any level of genotoxic carcinogens might pose a health risk to consumers. However, it has also been noted that the limit of 50 $\mathrm{ngkg}^{-1}$ may not be achievable in some regions of the world and the limit of $500 \mathrm{ngkg}^{-1}$ is adequate for the protection of consumer health. To achieve the strict limit of $50 \mathrm{ngkg}^{-1}$, the maximum allowable concentrations of $\mathrm{AFB}_{1}$ in dairy feeds and rations is $5,000 \mathrm{ngkg}^{-1}$ (European Commission, 2011; Kunsagi et al., 2011; Alvarado et al., 2017). The current Iranian standards for $\mathrm{AFM}_{1}$ and $\mathrm{AFB}_{1}$ are $100 \mathrm{ngkg}^{-1}$ and 5,000 ngkg ${ }^{-1}$, respectively (ISIRI, 2016; 2020).

Recent Iranian surveys and meta-analyses (Mashak et al., 2016; Tajik et al., 2016; Hamzeh Pour et al., 2020; Khaneghahi-Abyaneh, et al., 2019) have shown that the level of $\mathrm{AFM}_{1}$ in raw and pasteurized milk may be well below the ISIRI limit $\left(100 \mathrm{ngkg}^{-1}\right)$ but may exceed the limit of 50 ngkg ${ }^{-1}$, and few samples may exceed the limit of $500 \mathrm{ngkg}^{-1}$. Also, some studies have assessed AFB 1 contamination of feeds including corn silage, alfalfa hay, straw, barley, wheat bran, beet pulp, canola meal and grain mix and have reported various prevalence of contamination (19.2\%: Beheshti and Asadi, 2014; 82.5\%: Bahrami et al, 2016; $100 \%$ : Rezaei et al, 2014). In the study of Bahrami et al. (2016), $65 \%$ of corn silage and $10 \%$ of straw samples had contaminations above 5,000 ngkg ${ }^{-1}$. Seasonal differences in the levels of AFM $_{1}$ (Khaneghahi-Abyaneh, et al., 2019; Mozaffari Nejad et al., 2019; De Roma et al., 2017; Mahmoudi and Norian, 2015; Bahrami et al., 2016; Heshmati and Milani, 2010; Kamkar, 2005) and AFB 1 in feeds (Bahrami et al., 2016; Dimitrieska-Stojković et al., 2015; Mahmoudi and Norian, 2015; Simas et al., 2007) have been reported with higher levels in cold seasons.

The AFM ${ }_{1}$ contamination of milk and its seasonal variations would be best controlled if the level of $\mathrm{AFB}_{1}$ in the concurrent rations and feed ingredients are defined. In the present study, performed in a number of large dairy operations during cold and warm seasons, the level and the seasonal changes of $A_{F} M_{1}$ were screened in bulk milk to assess the probable hazard. The $\mathrm{AFB}_{1}$ contaminations of the concurrent rations and separate feed ingredients were also assessed to explain the most critical dietary contaminants in the studied farms.

\section{Materials And Methods}

\section{Farms and samplings}

The study was done in twelve selected dairy farms in Qazvin province, north-west, Iran, during winter and summer, 2019. The criteria for selecting the farms were having distinct role in the provision of the total milk produced in the province, being scattered in various parts of the province, willing for cooperation and provision of the requested data. The farms owned about 17,700 milking cows during the study period and produced about $40 \%$ of the total daily raw milk in the province (Cooperative Organization of Dairy Farms, Qazvin province; personal communication). The average milk production of the farms within the year of study was $30.5 \mathrm{~kg} / \mathrm{cow} /$ day. In each season, in a 30-day period, bulk milk and rations were sampled. The bulk milk produced within the last 24 hours was sampled on days 1, 15 and 30 ( $n=36 /$ season, $100 \mathrm{ml}$ in screw tap sterile bottles). Samples were taken according to the procedure No 87-44-05 communicated by Iranian Veterinary Organization (IVO, 2005) from a depth of about $20 \mathrm{~cm}$ of the top surface in the milk tank. The stirrers were working for about 10 minutes before sampling. The samples were transferred cool to the laboratory and were kept frozen $\left(-19^{\circ} \mathrm{C}\right)$ for about 30 days before the measurement of $A F M_{1}$. 
Sampling of feeds was preformed 2 days before the first and the last bulk milk sampling. The total mixed rations (TMR) of the milking cows were sampled two times (30 days apart) from the mangers immediately after the distribution of the morning meal according the procedure described by Robinson and Meyer (2010) ( $n=24 /$ season; about $0.5 \mathrm{~kg}$ each). The ingredients of the rations (Table 1 ) were also sampled ( $\mathrm{n}=230$, both seasons; about $0.5 \mathrm{~kg}$ each) in zipped nylon bags (Procedure No 84-44-06; IVO, 2008). The wet samples (TMRs, corn silage and wet beet pulp) were transferred cool to the laboratory and were oven dried at 50 to $70^{\circ} \mathrm{C}$ (24 hours; Memmert, UM200, Germany). The samples were powdered in a mill carefully cleaned for each sample, were heated at $105^{\circ} \mathrm{C}$ for 24 hours and $\mathrm{AFB}_{1}$ was measured in dry matter. Completion of sampling, transferring and collecting the required data (e.g. the formulations of the rations) all were performed by one trained person and took about two months in each season. Storing and preparation of the samples and measurement of $\mathrm{AFM}_{1}$ and $\mathrm{AFB}_{1}$ on milk and feed samples were done at the central diagnostic laboratory of the Veterinary Organization, Qazvin, Iran.

Measurement of $\mathrm{AFM}_{1}$ in milk and $\mathrm{AFB}_{1}$ in feeds

The concentration of $\mathrm{AFM}_{1}$ in milk and $\mathrm{AFB}_{1}$ in feeds were determined using direct competitive ELISA kits (Bioassay Technology Laboratory, China). The kits were stored at $2-8^{\circ} \mathrm{C}$, and before using they were put at room temperature $\left(25 \pm 2^{\circ} \mathrm{C}\right)$ for two hours. All steps of the experiments were done avoiding direct sun light.

\section{$\mathrm{AFM}_{1}$}

The frozen milk samples were thawed at room temperature $\left(25 \pm 2^{\circ} \mathrm{C}\right)$ and after reaching to thermal equilibrium, they were centrifuged at $3000 \mathrm{~g}$ for $10 \mathrm{minutes}$. The skimmed milk was used for $\mathrm{AFM}_{1}$ determination. Ten $\mathrm{ml}$ of each sample was mixed with $20 \mathrm{ml}$ of $70 \%$ ethanol in a screw-tap vial and was shaken vigorously for 3 minutes. Then, the mixtures were centrifuged at $3000 \mathrm{~g}$ and $100 \mu \mathrm{L}$ of the supernatant liquid was mixed with $400 \mu \mathrm{L}$ of the diluent solution. Using the provided microplate, $50 \mu \mathrm{L}$ of the standard solutions $\left(0.1,0.3 .0 .9,2.7\right.$ and $8.1 \mathrm{ppb}$ of $\left.\mathrm{AFM}_{1}\right)$ were put in the corresponding wells for preparing the standard curve. The same amounts of the prepared samples were put in the other wells. To all wells, was added $50 \mu \mathrm{L}$ of anti-AFM $\mathrm{M}_{1}$ conjugate antibody. The plate was shaken gently for a few seconds and then was warmed in $37^{\circ} \mathrm{C}$ bath for 30 minutes. The liquid in the holes was emptied and the microplate was washed 5 times (Bio-Tek ELx50, Bio-Tek Instruments, USA) using the washing solution. To all holes, were added 50 $\mu \mathrm{L}$ of color solutions A and B and the plate was put in $37^{\circ} \mathrm{C}$ bath for 10 minutes (formation of blue color). Finally, $50 \mu \mathrm{L}$ of the stop solution was added to each hole to terminate the reaction (formation of yellow color). The absorbance of the samples was determined at 450nm within 5 minutes (Bio-Tek ELx800, Bio-Tek Instruments, USA). The absorbance value was inversely related to the concentration of $\mathrm{AFM}_{1}$. The analytical range of the $\mathrm{AFM}_{1} \mathrm{kit}$ was $1-81 \mathrm{ngkg}^{-1}$. The values were reported as ngkg ${ }^{-1}$ ( $\mathrm{ppb}$ ).

\section{$\mathrm{AFB}_{1}$}

Five grams of each powdered feed sample was mixed with $25 \mathrm{ml}$ of $70 \%$ ethanol in a screw-tap vial, was left at room temperature overnight and then was shaken vigorously for 10 minutes. Then, the samples were centrifuged at $3000 \mathrm{~g}$ and the supernatant liquids were filtered on Whatman 1 papers. Exactly 100 $\mu \mathrm{L}$ of the filtered liquid was mixed with $400 \mu \mathrm{L}$ of the diluent solution. The other steps for measuring $\mathrm{AFB}_{1}$ were the same as described for $\mathrm{AFM}_{1}$. The standard curves were prepared using the standard solutions of $0,0.05,0.15,0.45,1.35$ and $4.05 \mathrm{ppb}$ of $\mathrm{AFB}_{1}$. The analytical range of the $\mathrm{AFB}_{1}$ kit was $1.25-101.25$ $\mathrm{ngkg}^{-1}$. The values were reported as $\mathrm{ngkg}^{-1}(\mathrm{ppb})$. The AFB ${ }_{1}$ concentrations in TMRs (Table 1 ) were reported as measures calculated from the weights and $\mathrm{AFB}_{1}$ contaminations of separate ingredients (calculated TMR) and also as the contaminations of the samples taken from the bunks (bunk TMR).

\section{Statistical analysis}

The results were statistically studied using SPSS software (version 24, Illinois, USA). For aflatoxin values out of the analytical range of the corresponding kit (1-81 $\mathrm{ngkg}^{-1}$ for $\mathrm{AFM}_{1} ; 1.25-10.25 \mathrm{ngkg}^{-1}$ for $\mathrm{AFB}_{1}$ ) the lower and the upper limits were substituted as the lowest and the highest detected values, respectively. Descriptive statistics were presented as means and standard deviations. The aflatoxin levels in milk and bunk TMR samples were classified into two relatively equal-sized groups based on their medians. Association of the aflatoxin with season was evaluated using generalized estimating equations (GEE) with binomial distribution and logit function. Farm was introduced into the model as subject effect and season as well as number of samplings in each season were considered as repeated effects to account for the dependence between measurements. Separate models were constructed for $\mathrm{AFM}_{1}$ and $\mathrm{AFB}_{1}$ in milk and bunk TMR, respectively. The aflatoxin concentration in the calculated TMR and ration ingredients as well as ration DM (bunk and calculated, see below) of winter and summer were compared using linear mixed models. Farm was considered as random effect, and season and number of samples as fixed effects in the model. For ration ingredients, an additional fixed effect for type of feeds was introduced into the model followed by multiple comparisons with Bonferroni adjustment. The relationships between milk $\mathrm{AFM}_{1}$ with $\mathrm{AFB}_{1}$ in the TMR (bunk and calculated) and the separate ration ingredients were assessed by Spearman's rho correlation test. The P-value less than 0.05 was considered significant. 


\begin{tabular}{|c|c|c|c|c|c|c|c|c|c|c|c|c|c|}
\hline \multirow[t]{3}{*}{ Farm } & \multirow[t]{3}{*}{ Ration ingredient } & \multicolumn{4}{|c|}{ Feed weight in the ration (kg DM) } & \multicolumn{4}{|c|}{ Feed $\mathrm{AFB}_{1}(\mathrm{ng} / \mathrm{kg} \mathrm{DM})$} & \multicolumn{4}{|c|}{ Total $\mathrm{AFB}_{1}(\mathrm{ng})$ in the whole ration $\mathrm{DM}$} \\
\hline & & \multicolumn{2}{|l|}{ Winter } & \multicolumn{2}{|c|}{ Summer } & \multicolumn{2}{|l|}{ Winter } & \multicolumn{2}{|c|}{ Summer } & \multicolumn{2}{|l|}{ Winter } & \multicolumn{2}{|l|}{ Summer } \\
\hline & & 2 & 1 & 2 & 1 & 2 & 1 & 2 & 1 & 2 & 1 & 2 & \\
\hline \multirow[t]{5}{*}{1} & Alf. hay & 2.09 & 2.07 & 4.46 & 4.24 & 30.69 & 78.95 & 35.31 & 4.84 & 64.17 & 163.26 & 157.41 & 20.53 \\
\hline & Corn sil. & 13.15 & 4.97 & 3.80 & 4.33 & 3.03 & 1.94 & 36.15 & 10.08 & 39.84 & 9.63 & 137.51 & 43.61 \\
\hline & BP-dry & - & - & - & - & 12.58 & 7.35 & 16.42 & 7.98 & - & - & - & - \\
\hline & BP-wet & 2.37 & 1.17 & - & 1.39 & 2.37 & 1.25 & - & 101.25 & 5.61 & 1.46 & - & 140.71 \\
\hline & Grain mix & 13.19 & 13.28 & 12.71 & 12.67 & 2.39 & 1.25 & 27.38 & 101.25 & 31.53 & 16.60 & 347.90 & 1283.26 \\
\hline \multicolumn{2}{|c|}{ TMR (calculated) } & 30.8 & 21.49 & 20.97 & 22.64 & & & & & 141.14 & 190.96 & 642.86 & 1488.11 \\
\hline \multicolumn{2}{|c|}{ TMR (bunk) } & 14.17 & 21.84 & 21.22 & 22.11 & 1.25 & 24.35 & 52.49 & 101.25 & 17.72 & 531.84 & 1113.62 & 2238.80 \\
\hline \multirow[t]{5}{*}{2} & Alf. hay & 2.76 & 2.71 & 2.83 & 2.85 & 1.87 & 101.25 & 101.25 & 101.25 & 5.16 & 274.04 & 287.01 & 288.48 \\
\hline & Corn sil. & 4.37 & 5.89 & 4.73 & 4.77 & 1.25 & 42.27 & 67.79 & 23.52 & 5.46 & 248.85 & 320.35 & 112.13 \\
\hline & BP-dry & 0.64 & 0.63 & 0.92 & 0.75 & 18.88 & 19.49 & 101.25 & 1.25 & 12.11 & 12.34 & 93.56 & 0.94 \\
\hline & BP-wet & - & - & - & - & 2.00 & 91.98 & - & 5.48 & - & - & - & - \\
\hline & Grain mix & 15.18 & 15.50 & 15.40 & 15.61 & 12.68 & 4.77 & 65.12 & 16.36 & 192.44 & 73.95 & 1002.83 & 255.45 \\
\hline \multicolumn{2}{|c|}{ TMR (calculated) } & 22.95 & 24.73 & 23.88 & 23.98 & & & & & 215.23 & 609.19 & 1703.67 & 656.99 \\
\hline \multicolumn{2}{|c|}{ TMR (bunk) } & 32.79 & 20.36 & 18.51 & 14.91 & 28.63 & 34.39 & 240.67 & 81.20 & 938.19 & 700.09 & 1874.22 & 1210.67 \\
\hline \multirow[t]{5}{*}{3} & Alf. hay & 1.84 & 1.86 & 2.60 & 2.58 & 6.11 & 24.71 & 101.25 & 13.48 & 11.27 & 45.95 & 263.07 & 34.81 \\
\hline & Corn sil. & 7.74 & 9.30 & 6.68 & 4.47 & 20.69 & 1.25 & 92.84 & 101.25 & 160.08 & 11.63 & 620.40 & 452.44 \\
\hline & BP-dry & 1.35 & 1.38 & 0.94 & 0.95 & 14.77 & 27.29 & 10.42 & 9.52 & 19.91 & 37.78 & 9.81 & 9 \\
\hline & BP-wet & - & - & - & - & 15.23 & 48.45 & 21.86 & 91.42 & - & - & - & - \\
\hline & Grain mix & 15.38 & 15.48 & 14.08 & 13.71 & 94.80 & 62.77 & 27.22 & 7.34 & 1457.69 & 971.37 & 390.39 & 100.61 \\
\hline \multicolumn{2}{|c|}{ TMR (calculated) } & 26.31 & 28.02 & 24.31 & 21.70 & & & & & 1648.95 & 1066.73 & 1283.68 & 596.86 \\
\hline \multicolumn{2}{|c|}{ TMR (bunk) } & 27.64 & 26.80 & 20.04 & 22.84 & 2.73 & 5.23 & 10.51 & 13.29 & 75.47 & 140.15 & 210.63 & 303.57 \\
\hline \multirow[t]{5}{*}{4} & Alf. hay & 1.81 & 1.83 & 2.98 & 3.04 & 17.20 & 101.25 & 101.25 & 11.63 & 31.21 & 184.82 & 301.26 & 35.37 \\
\hline & Corn sil. & 5.91 & 4.35 & 4.81 & 5.03 & 1.99 & 53.42 & 50.33 & 101.25 & 11.75 & 232.46 & 242.20 & 508.92 \\
\hline & BP-dry & 1.82 & 1.84 & - & - & 1.83 & 49.23 & - & 101.25 & 3.34 & 90.41 & - & - \\
\hline & BP-wet & - & - & - & - & - & - & - & - & - & - & - & - \\
\hline & Grain mix & 15.33 & 15.33 & 14.32 & 14.55 & 24.84 & 13.05 & 52.49 & 101.25 & 380.85 & 202.62 & 751.76 & 1473.17 \\
\hline TMR & alculated) & 24.87 & 23.35 & 22.11 & 22.62 & & & & & 727.16 & 710.31 & 1295.21 & 2017.47 \\
\hline TMR & unk) & 39.45 & 19.59 & 19.03 & 22.15 & 33.24 & 3.93 & 99.33 & 101.25 & 1311.15 & 76.97 & 1889.81 & 2243.09 \\
\hline 5 & Alf. hay & 1.82 & 1.87 & 1.82 & 1.90 & 21.28 & 101.25 & 92.84 & 101.25 & 38.83 & 188.85 & 169.04 & 192.27 \\
\hline & Corn sil. & 11.12 & 6.94 & 9.13 & 6.17 & 15.79 & 4.34 & 51.94 & 6.25 & 175.64 & 30.10 & 474.30 & 38.56 \\
\hline & BP-dry & 0.55 & 0.56 & 0.55 & 0.59 & 1.39 & 21.07 & 57.04 & 75.71 & 0.77 & 11.78 & 31.43 & 44.68 \\
\hline & BP-wet & - & - & - & - & 3.12 & 1.25 & 80.22 & 9.69 & - & - & - & - \\
\hline & Grain mix & 15.42 & 15.49 & 15.41 & 15.25 & 21.15 & 7.10 & 68.47 & 5.20 & 326.08 & 110.01 & 1055.39 & 79.29 \\
\hline TMR & alculated) & 28.91 & 24.86 & 26.91 & 23.91 & & & & & 541.32 & 340.74 & 1730.16 & 354.81 \\
\hline TMR & unk) & 30.26 & 32.34 & 22.28 & 24.77 & 2.74 & 22.63 & 70.56 & 69.87 & 82.91 & 731.89 & 1572.38 & 1730.56 \\
\hline 6 & Alf. hay & 4.11 & 4.17 & 4.49 & 4.53 & 4.34 & 16.01 & 20.06 & 101.25 & 17.86 & 66.69 & 90.14 & 459.03 \\
\hline & Corn sil. & 4.69 & 8.11 & 4.80 & 4.58 & 1.25 & 5.05 & 43.76 & 101.25 & 5.86 & 40.97 & 209.84 & 463.64 \\
\hline & BP-dry & - & - & - & - & 6.40 & 22.31 & 9.43 & 6.93 & - & - & - & - \\
\hline
\end{tabular}




\begin{tabular}{|c|c|c|c|c|c|c|c|c|c|c|c|c|}
\hline \multicolumn{13}{|c|}{$\begin{array}{l}\text { Table } 1 \\
\text { Weight, } \mathrm{AFB}_{1} \text { concentration and the contribution of ration ingredients in total contamination of the ration }\end{array}$} \\
\hline BP-wet & 1.01 & 0.99 & 1.19 & 1.02 & 1.25 & 1.54 & 97.43 & 5.30 & 1.27 & 1.53 & 115.92 & 5.40 \\
\hline Grain mix & 13.18 & 13.39 & 15.44 & 15.38 & 1.77 & 4.96 & 46.20 & 13.69 & 23.33 & 66.40 & 713.44 & 210.56 \\
\hline TMR (calculated) & 22.99 & 26.66 & 25.92 & 25.51 & & & & & $48 . .32$ & 175.59 & 1129.34 & 1138.64 \\
\hline TMR (bunk) & 33.08 & 32.60 & 27.36 & 25.20 & 18.58 & 50.04 & 1.25 & 101.25 & 614.58 & 1631.05 & 34.19 & 2551.20 \\
\hline Alf. hay & 1.83 & 1.86 & 1.65 & 1.64 & 101.25 & 3.22 & 1.25 & 101.25 & 187.75 & 5.99 & 2.06 & 166.05 \\
\hline Corn sil. & 5.46 & 7.13 & 4.86 & 4.65 & 16.54 & 5.08 & 57.63 & 45.34 & 90.32 & 36.20 & 279.94 & 210.77 \\
\hline BP-dry & - & - & - & - & 1.25 & 20.63 & 40.96 & 9.77 & - & - & - & - \\
\hline BP-wet & 1.01 & 1.08 & 0.81 & 1.27 & 16.91 & 28.88 & 46.20 & 7.01 & 17.06 & 31.19 & 37.38 & 8.89 \\
\hline Grain mix & 15.39 & 15.69 & 15.47 & 15.49 & 34.16 & 11.06 & 8.70 & 101.25 & 325.55 & 173.49 & 134.57 & 1568.06 \\
\hline TMR (calculated) & 23.69 & 25.75 & 22.79 & 23.05 & & & & & 818.68 & 246.86 & 453.96 & 1953.76 \\
\hline TMR (bunk) & 26.22 & 29.75 & 17.89 & 19.85 & 25.13 & 1.25 & 58.83 & 101.25 & 658.91 & 37.18 & 1052.70 & 2009.71 \\
\hline Alf. hay & 1.14 & 1.16 & 1.89 & 1.82 & 1.25 & 60.84 & 84.25 & 101.25 & 1.43 & 70.70 & 159.54 & 184.05 \\
\hline Corn sil. & 9.39 & 6.40 & 7.01 & 7.18 & 4.56 & 25.08 & 70.56 & 7.16 & 42.81 & 160.40 & 494.80 & 51.41 \\
\hline BP-dry & - & - & - & - & 2.24 & 2.89 & 101.25 & 85.12 & - & - & - & - \\
\hline BP-wet & 1.09 & 1.03 & 1.22 & 1.44 & 3.16 & 2.21 & 56.46 & 20.87 & 3.45 & 2.27 & 68.65 & 30.05 \\
\hline Grain mix & 13.74 & 14.08 & 16.02 & 16.02 & 1.25 & 4.81 & 33.69 & 60.08 & 17.18 & 67.73 & 539.81 & 962.66 \\
\hline TMR (calculated) & 25.36 & 22.67 & 26.14 & 26.46 & & & & & 64.87 & 301.08 & 1262.80 & 1228.18 \\
\hline TMR (bunk) & 33.23 & 25.62 & 25.72 & 23.21 & 22.70 & 1.25 & 83.42 & 99.82 & 754.29 & 32.02 & 2145.75 & 2316.74 \\
\hline Alf. hay & 2.40 & 2.37 & 2.32 & 2.36 & 16.07 & 79.86 & 98.37 & 101.25 & 38.58 & 189.20 & 228.46 & 238.87 \\
\hline Corn sil. & 7.96 & 5.08 & 4.95 & 5.63 & 2.47 & 1.25 & 5.72 & 3.56 & 19.66 & 6.36 & 28.30 & 20.04 \\
\hline BP-dry & 0.92 & 0.91 & 0.90 & 0.93 & 4.11 & 11.05 & 28.42 & 1.25 & 3.76 & 10.03 & 25.66 & 1.17 \\
\hline BP-wet & - & - & - & - & - & - & - & - & - & - & - & - \\
\hline Grain mix & 13.15 & 13.23 & 12.68 & 12.70 & 5.46 & 5.01 & 40.50 & 8.68 & 71.82 & 66.30 & 513.42 & 110.26 \\
\hline TMR (calculated) & 24.43 & 21.59 & 20.85 & 21.62 & & & & & 133.82 & 271.87 & 795.85 & 370.33 \\
\hline TMR (bunk) & 25.26 & 15.85 & 20.47 & 19.15 & 17.78 & 1.25 & 2.98 & 5.83 & 449.18 & 19.81 & 61.01 & 111.63 \\
\hline Alf. hay & & & & & 2.35 & 101.25 & 101.25 & 20.39 & & & & \\
\hline Corn sil. & & & & & 4.49 & 21.99 & 74.15 & 78.48 & & & & \\
\hline BP-dry & & & & & 3.95 & 45.51 & 89.31 & 9.94 & & & & \\
\hline BP-wet & & & & & 2.46 & 1.25 & 71.97 & 86.40 & & & & \\
\hline Grain mix & & & & & 1.25 & 16.50 & 65.12 & 101.25 & & & & \\
\hline \multicolumn{13}{|l|}{ TMR (calculated) } \\
\hline TMR (bunk) & & & & & 3.92 & 1.25 & 96.49 & 101.25 & & & & \\
\hline Alf. hay & & & & & 101.25 & 1.25 & 1.25 & 101.25 & & & & \\
\hline Corn sil. & & & & & 22.80 & 57.99 & 101.25 & 101.25 & & & & \\
\hline BP-dry & & & & & 2.76 & 20.19 & 101.25 & 2.23 & & & & \\
\hline BP-wet & & & & & 2.33 & 29.37 & 101.25 & 25.74 & & & & \\
\hline Grain mix & & & & & 4.16 & 10.92 & 35.73 & 8.06 & & & & \\
\hline \multicolumn{13}{|l|}{ TMR (calculated) } \\
\hline TMR (bunk) & & & & & 23.73 & 71.47 & 81.81 & 101.25 & & & & \\
\hline Alf. hay & 3.13 & 3.26 & 2.80 & 2.85 & 25.42 & 35.90 & 83.42 & 1.25 & 79.65 & 117.11 & 233.57 & 3.56 \\
\hline Corn sil. & 4.37 & 10.13 & 4.69 & 3.45 & 14.95 & 30.67 & 35.31 & 14.02 & 65.30 & 310.63 & 165.53 & 48.31 \\
\hline
\end{tabular}




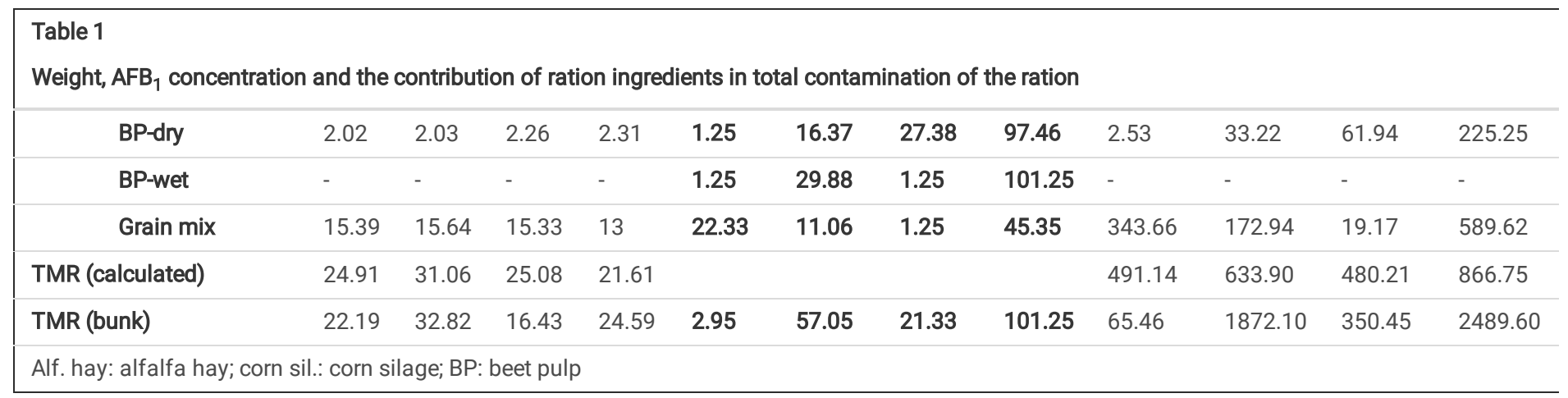

\section{Results}

Aflatoxin $\mathrm{M}_{1}$ in milk

Aflatoxin $M_{1}$ was detected in all bulk milk samples ( $n=72 ; 100 \%$ ) ranging from 2.03 to $>81 \mathrm{ngkg}^{-1}$ (Table 2). The average seasonal contaminations (considering $81 \mathrm{ngkg}^{-1}$ as the highest detected value) were $61.25 \pm 28.91 \mathrm{ngkg}^{-1}$ in winter and $54.20 \pm 25.51 \mathrm{ngkg}^{-1}$ in summer $(P=0.279)$; and the median was $70 \mathrm{ngkg}^{-1}$. The frequencies of the samples with $\mathrm{AFM}_{1}$ levels below and above the median are shown in Tabla 2.

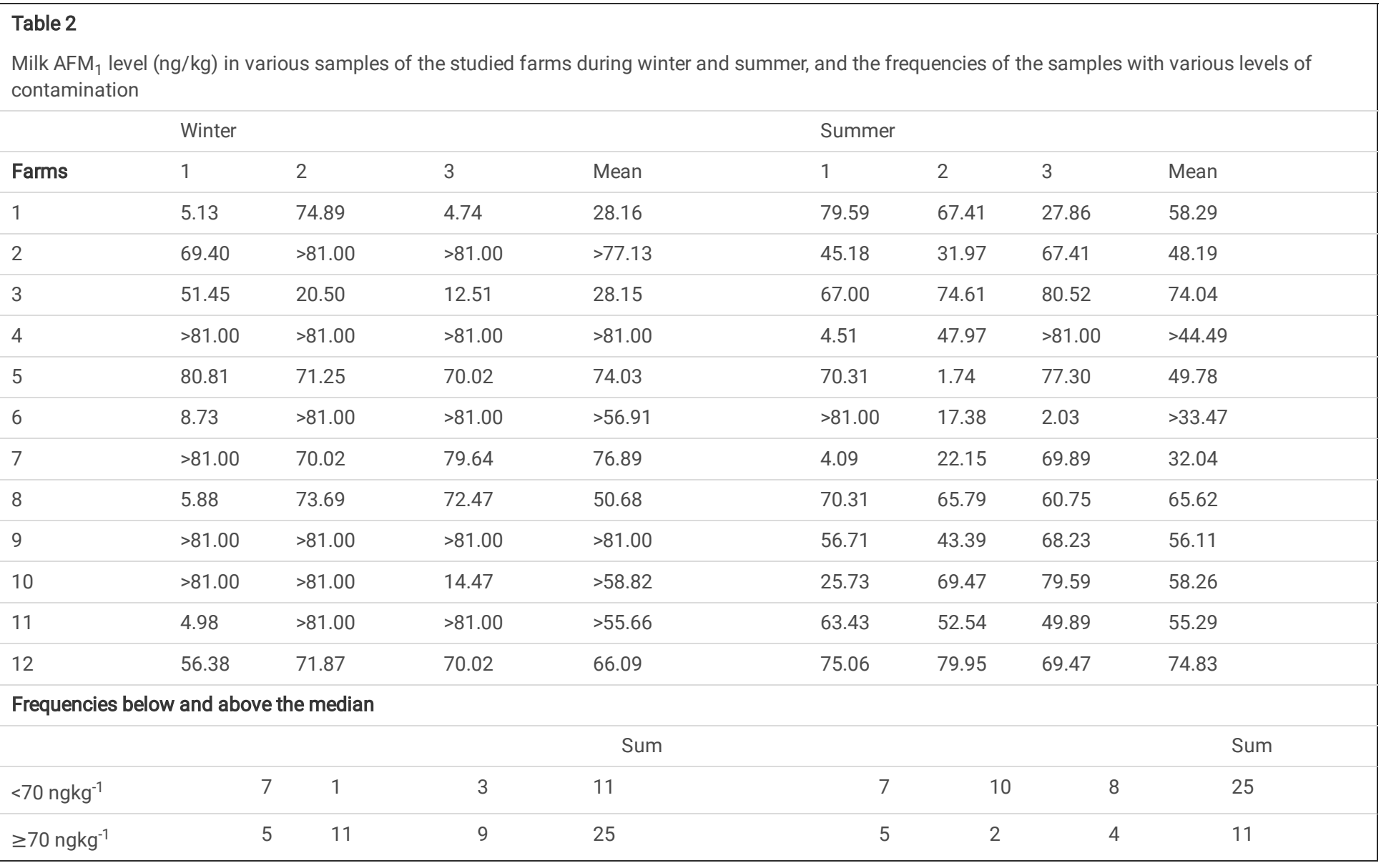

Figure 1 shows that $29 \%$ of samples (21 out of 72 ) had $\mathrm{AFM}_{1}$ levels below $50 \mathrm{ngkg}^{-1}, 47 \%$ (34 samples) had contaminations between $50-81$ ngkg ${ }^{-1}$ and $24 \%$ (17 samples) had contaminations above $81 \mathrm{ngkg}^{-1}$. The contamination of the latter category could potentially be below or above the Iranian standard (100 ngkg ${ }^{-1}$ ). Overall, $76 \%$ of bulk milk samples (56 out of 72 ) had aflatoxin levels less than $81 \mathrm{ngkg}^{-1}$.

Regarding the seasonal variations, contaminations $>81 \mathrm{ngkg}^{-1}$ were more frequent in winter ( $42 \%$ in winter vs. $6 \%$ in summer; Figure 2$)$. Examining the median $\mathrm{AFM}_{1}$ contamination, $69 \%$ of winter samples $(\mathrm{n}=25 / 36)$ had contaminations above $70 \mathrm{ngkg}^{-1}$ and a reverse result was detected in summer (Table 2$)$. In fact, the results of GEE for association of milk aflatoxin with season showed the chance of contaminations above $70 \mathrm{ngkg}^{-1}$ was 5.33 times higher in winter than in summer $(\mathrm{OR}=5.33, \mathrm{P}=0.007)$.

Aflatoxin $B_{1}$ in Feed ingredients and TMRs: 
The weights $(\mathrm{DM}), \mathrm{AFB}_{1}$ contamination and the share of each ingredient in the whole ration contamination, and total $\mathrm{AFB}_{1}$ content of the $\mathrm{TMR}$ are shown in Table 1. The data on TMR were calculated from the weight and AFB 1 contamination of separate ingredients (calculated TMR). The contamination of the TMR samples taken from the bunks are shown in separate rows (bunk TMR). Two farms did not provide the ration details and so, only the contamination of their feed samples is shown.

The average $\mathrm{AFB}_{1}$ contaminations of separate ration ingredients $(\mathrm{n}=230)$ and the frequencies of non-detectable $\left(<1.25 \mathrm{ngkg}^{-1}\right)$ and high $\left(>101.25 \mathrm{ngkg}{ }^{-1}\right)$ contaminations are depicted in Table 3. All samples had significantly higher contaminations in summer compared with winter. Alfalfa hay had the highest averages in both seasons $(P<0.05)$. It also showed the most frequent values above $101.25 \mathrm{ngkg}^{-1} \mathrm{DM}$. Twenty-three samples (10\%) had non-detectable levels of $\mathrm{AFB}_{1}$ and 32 samples (13.9\%) had values above $101.25 \mathrm{ngkg}^{-1}$. The non-detectable values were mostly seen in winter ( $\left.\mathrm{n}=17 / 23\right)$, while the values above $101.25 \mathrm{ngkg}^{-1}$ were mainly detected in summer $(\mathrm{n}=27 / 32)$.

\section{Table 3}

Aflatoxin concentration (mean $\pm S D)^{1}$ in the ration ingredients in the studied farms

Feeds Number of Samples Aflatoxin concentration $(\mathrm{ng} / \mathrm{kg} \mathrm{DM})$

Winter Summer Winter $\quad$ Summer $\quad$ Mean $\quad$ Frequencies

$<1.25>101.25$

\begin{tabular}{|c|c|c|c|c|c|c|c|}
\hline \multicolumn{8}{|l|}{ Ingredients } \\
\hline Alfalfa hay & 24 & 24 & $39.80 \pm 38.92^{a}$ & $65.92 \pm 43.36^{\mathrm{a}^{*}}$ & $52.86 \pm 42.85^{a}$ & 5 & 16 \\
\hline Corn silage & 24 & 24 & $15.01 \pm 16.79^{b}$ & $53.37 \pm 35.40^{b^{*}}$ & $34.19 \pm 33.59^{b}$ & 4 & 5 \\
\hline BP-dry & 24 & 23 & $13.94 \pm 13.19^{b}$ & $43.11 \pm 40.49^{b^{*}}$ & $28.22 \pm 32.99 b$ & 4 & 4 \\
\hline BP-wet & 20 & 19 & $14.31 \pm 22.80^{b}$ & $51.73 \pm 39.58^{b^{*}}$ & $32.03 \pm 36.68^{b}$ & 6 & 3 \\
\hline Grain mix & 24 & 24 & $15.81 \pm 21.70^{b}$ & $43.42 \pm 33.49^{b^{*}}$ & $29.62 \pm 31.21^{b}$ & 4 & 4 \\
\hline Sum & 116 & 114 & & & & 23 & 32 \\
\hline
\end{tabular}

1: assuming that the values of 1.25 and $101.25 \mathrm{ngkg}^{-1}$ (the lower and the upper limits of the experimental kit) were the lowest and the highest contamination rates; a, b: different letters indicate to significant differences in the columns $(P<0.05)$, *: Asterix indicate to significant differences in rows $(\mathrm{P}<0.05)$; BP: beet pulp; DM: dry matter

The average $A F B_{1}$ content of the TMRs (Table 4) was higher in summer than in winter for both calculated $(P<0.001)$ and bunk $(P=0.002)$ TMRs, so that each cow could potentially consume at least 2.4-2.6 times more aflatoxin in summer. The bunk samples were contaminated higher than the calculated TMRs.

Among the bunk TMRs ( $\mathrm{n}=48$ ), 6 samples had non-detectable values ( 5 in winter) and 7 samples had levels above 101.25 ngkg-1 (all in summer; Table 4 ). The median contamination of bunk TMRs was $716 \mathrm{ngkg}^{-1}$ and the GEE results showed that the chance of contaminations above median was 5.57 times higher in summer than in winter $(\mathrm{OR}=5.57, \mathrm{P}=0.002)$. Seventy percent of the bunk TMR samples had contaminations above the median in summer (Figure 3$)$. 


\section{Table 4}

The concentration ( $\mathrm{ngkg}^{-1} \mathrm{DM}$ ) and the total daily content (ng) of $\mathrm{AFB}_{1}$ (mean $\pm \mathrm{SD}$ )* in TMRs of the studied farms

\begin{tabular}{|c|c|c|c|c|c|c|c|c|}
\hline & \multicolumn{6}{|c|}{ Season and sampling } & \multicolumn{2}{|l|}{ Averages } \\
\hline TMR type & Winter1 & Winter2 & Summer1 & Summer2 & Min & Max & Winter & Summer \\
\hline \multicolumn{9}{|l|}{ Calculated } \\
\hline \multirow[t]{2}{*}{$\begin{array}{l}\text { Ration DM } \\
\text { (kg) }\end{array}$} & $\begin{array}{l}25.53 \pm 2.54 \\
(n=10)\end{array}$ & $\begin{array}{l}25.04 \pm 3.00 \\
(n=10)\end{array}$ & $\begin{array}{l}23.90 \pm 2.16 \\
(n=10)\end{array}$ & $\begin{array}{l}23.31 \pm 1.66 \\
(n=10)\end{array}$ & 20.85 & 31.06 & $\begin{array}{r}25.28 \\
\pm 2.72\end{array}$ & $\begin{array}{l}23.60 \\
\pm 1.90\end{array}$ \\
\hline & & & & & & & \multicolumn{2}{|l|}{$P=0.017$} \\
\hline \multirow[t]{2}{*}{$\begin{array}{l}\text { AFB1 } \\
\text { (ng/kg DM) }\end{array}$} & $\begin{array}{l}17.69 \pm 18.79^{a} \\
(n=10)\end{array}$ & $\begin{array}{l}17.80 \pm 10.30^{a} \\
(n=10)\end{array}$ & $\begin{array}{l}44.68 \pm 17.86^{b} \\
(n=10)\end{array}$ & $\begin{array}{l}44.78 \pm 26.44^{b} \\
(n=10)\end{array}$ & 2.10 & 89.20 & $\begin{array}{l}17.74 \\
\pm 14.74\end{array}$ & $\begin{array}{l}45.22 \\
\pm 21.97\end{array}$ \\
\hline & & & & & & & \multicolumn{2}{|l|}{$P<0.001$} \\
\hline $\begin{array}{l}\text { Daily AFB1 } \\
\text { intake (ng) }\end{array}$ & $\begin{array}{l}453.06 \pm 488.08^{a} \\
(n=10)\end{array}$ & $\begin{array}{l}454.72 \pm 289.94^{a} \\
(n=10)\end{array}$ & $\begin{array}{l}1077.80 \pm 466.52^{b} \\
(n=10)\end{array}$ & $\begin{array}{l}1067.20 \pm 607.36^{b} \\
(n=10)\end{array}$ & 48.32 & 2017.47 & $\begin{array}{l}453.89 \\
\pm 390.72\end{array}$ & $1072.50 \pm 527.12$ \\
\hline
\end{tabular}

\section{Bunk}

\begin{tabular}{|c|c|c|c|c|c|c|c|c|}
\hline $\begin{array}{l}\text { Ration DM } \\
(\mathrm{kg})\end{array}$ & $\begin{array}{l}28.43 \pm 7.04 \\
(n=10)\end{array}$ & $\begin{array}{l}25.76 \pm 6.13 \\
(n=10)\end{array}$ & $\begin{array}{l}20.90 \pm 3.43 \\
(n=10)\end{array}$ & $\begin{array}{l}21.88 \pm 3.16 \\
(n=10)\end{array}$ & 14.17 & 39.45 & $\begin{array}{l}27.09 \\
\pm 6.57\end{array}$ & $\begin{array}{l}21.39 \\
\pm 3.25\end{array}$ \\
\hline & & & & & & & $P=0.003$ & \\
\hline \multirow[t]{2}{*}{$\begin{array}{l}\text { AFB1 (ng/kg } \\
\text { DM) }\end{array}$} & $\begin{array}{l}15.28 \pm 11.81^{a} \\
(n=12)\end{array}$ & $\begin{array}{l}22.84 \pm 25.12^{a} \\
(n=12)\end{array}$ & $\begin{array}{l}56.69 \pm 38.48^{b} \\
(n=12)\end{array}$ & $\begin{array}{l}81.56 \pm 35.14^{c} \\
(n=12)\end{array}$ & 1.25 & 101.25 & $\begin{array}{l}19.06 \\
\pm 19.58\end{array}$ & $\begin{array}{l}69.13 \\
\pm 38.21\end{array}$ \\
\hline & & & & & & & $P<0.001$ & \\
\hline $\begin{array}{l}\text { Daily AFB1 } \\
\text { intake (ng) }\end{array}$ & $\begin{array}{l}496.79 \pm 438.57^{a} \\
(n=10)\end{array}$ & $\begin{array}{l}577.31 \pm 680.62^{a} \\
(n=10)\end{array}$ & $\begin{array}{l}1013.50 \pm 820.72 \\
\text { a } \\
(n=10)\end{array}$ & $\begin{array}{l}1720.06 \pm 889.36^{b} \\
(n=10)\end{array}$ & 111.63 & 2551.20 & $\begin{array}{l}537.05 \\
\pm 558.79\end{array}$ & $\begin{array}{r}1375.50 \\
\pm 905.02\end{array}$ \\
\hline
\end{tabular}

\section{Frequencies $(n=48) * *$}

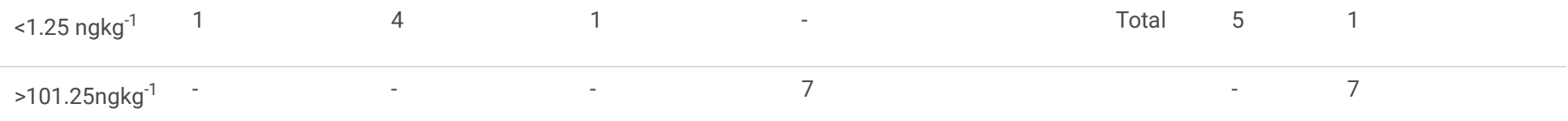

* Assuming that the values of 1.25 and $101.25 \mathrm{ngkg}-1$ (the lower and the upper limits of the experimental kit) were the lowest and the highest contamination rates; TMR: total mixed ration

** Excluding the two farms with incomplete data (totally 40 bunk TMR samples), 5 samples had non-detectable values (4 in winter) and 5 samples had levels above $101.25 \mathrm{ng} / \mathrm{kg}$ (all in summer)

Correlations

No correlation was detected in neither of seasons between the ration $\mathrm{AFB}_{1}$ and milk $\mathrm{AFM}_{1}$. There were some correlations between the $\mathrm{AFB} \mathrm{B}_{1}$ content of $\mathrm{TMR}_{\mathrm{B}}$ and the amount of $\mathrm{AFB}_{1}$ added to the ration by each ingredient (Table 5). The most frequent and the strongest correlations were seen for grain mix succeeded 
by corn silage, wet beet pulp and dry beet pulp, respectively. The AFB1 in alfalfa hay was correlated only with that of calculated TMR in the sum of both seasons, irrespective of its higher contaminations compared with the other ingredients. The correlations were less prominent for bunk TMRs.

The $\mathrm{AFB}_{1}$ levels in calculated and bunk TMRs were related in summer $(r=0.48, \mathrm{P}=0.033)$ and in the sum of both seasons $(r=0.43$, $\mathrm{P}=0.006)$, but not in winter $(r=-0.05, P=0.83)$.

\begin{tabular}{|c|c|c|c|c|c|c|}
\hline \multicolumn{7}{|l|}{ Table 5} \\
\hline \multicolumn{7}{|c|}{$\begin{array}{l}\text { Correlations ( } r \text { values) between the aflatoxin level in calculated or bunk TMR and the aflatoxin added to the ration by each ingredient (P values in } \\
\text { parentheses) }\end{array}$} \\
\hline & \multicolumn{3}{|c|}{ Calculated TMR } & \multicolumn{3}{|c|}{ Bunk TMR } \\
\hline & Winter & Summer & Both seasons & Winter & Summer & Both seasons \\
\hline \multirow[t]{2}{*}{ Alfalfa hay } & - & - & 0.31 & - & - & - \\
\hline & & & $(0.056)$ & & & \\
\hline \multirow[t]{2}{*}{ Corn silage } & 0.53 & 0.52 & 0.66 & - & - & 0.31 \\
\hline & $(0.016)$ & $(0.018)$ & $(0.001)$ & & & $(0.050)$ \\
\hline \multirow[t]{2}{*}{ Beet pulp-dry } & 0.61 & - & 0.42 & - & 0.67 & - \\
\hline & $(0.036)$ & & (0.053) & & (0.033) & \\
\hline \multirow[t]{2}{*}{ Beet pulp-wet } & - & - & 0.68 & - & - & - \\
\hline & & & $(0.005)$ & & & \\
\hline \multirow[t]{2}{*}{ Grain mix } & 0.82 & 0.91 & 0.90 & - & 0.45 & 0.38 \\
\hline & $(0.001)$ & $(0.001)$ & $(0.001)$ & & $(0.047)$ & (0.015) \\
\hline
\end{tabular}

\section{Discussion}

Aflatoxin M1 in milk

While all bulk milk samples ( $\mathrm{n}=72 ; 100 \%)$ showed contaminations with $\mathrm{AFM}_{1}, 55$ samples $(76 \%)$ had levels below $81 \mathrm{ngkg}^{-1}$. The remaining 17 samples $(24 \%)$ with higher contaminations could potentially be below or above the standard limit of $100 \mathrm{ngkg}^{-1}$. Twenty-one samples (29\%) were contaminated below 50 $\mathrm{ngkg}^{-1}$. The real contaminations could probably be lower than the detected values due to the method of prediction (ELISA). Less AFM 1 values are usually obtained with HPLC method compared with ELISA (Alvarado et al., 2017). A meta-analysis on Iranian studies (Khaneghahi-Abyaneh, et al., 2019) showed means of 59.19 and $35.23 \mathrm{ngkg}^{-1}$ with ELISA (55 studies; 9224 samples) and HPLC (18 studies; 2606 samples) methods, respectively. Some Iranian studies have reported $\mathrm{AFM}_{1}$ contamination in UHT and pasteurized milk. Mashak et al. (2016) detected $\mathrm{AFM}_{1}\left(15\right.$ to $140 \mathrm{ngkg}^{-1} ; \mathrm{HPLC}$ method) in all (100\%; $\mathrm{n}=30$ ) samples, $66.7 \%(n=20)$ having levels below $50 \mathrm{ngkg}^{-1}, 20 \%(\mathrm{n}=6)$ above $100 \mathrm{ngkg}^{-1}$ and none over $500 \mathrm{ngkg}^{-1}$. Tajik et al., (2016; ELISA method), found AFM $\left(75.8 \pm 9.2 \mathrm{ngkg}^{-1}\right)$ in $77.7 \%$ of samples ( $\left.\mathrm{n}=280 / 360\right), 36 \%(\mathrm{n}=130)$ showing levels below $50 \mathrm{ngkg}^{-1}$ and $2.2 \%$ ( $\left.\mathrm{n}=8\right)$ exceeding $500 \mathrm{ngkg}{ }^{-1}$. Heshmati and Milani (2010; ELISA method) found AFM $_{1}$ in $55.2 \%$ ( $\left.n=116 / 210\right)$ of samples, with $21.9 \%(n=46)$ having levels below $50 \mathrm{ngkg}^{-1}$ and none exceeding 500 $\mathrm{ngkg}^{-1}$. Based on a meta-analysis of 70 Iranian studies (Hamzeh-pour et al., 2020), 64\% of raw milk samples were contaminated to AFM (mean 39.7 ngkg $^{-1}$ ), $75 \%$ of which below $50 \mathrm{ngkg}^{-1}$ and $9 \%$ above $500 \mathrm{ngkg}^{-1}$. Contaminations above $50 \mathrm{ngkg}^{-1}$ have been reported from other countries (Škrbić et al., 2013 ; Tsakiris et al., 2013). Škrbić et al. (2013) reported a mean of $300 \mathrm{ngkg}^{-1} \mathrm{AFM}_{1}$ in commercial milk samples from Serbia.

Seasonal differences in milk AFM 1 levels have been reported with higher contaminations in cold seasons (Khaneghahi-Abyaneh, et al., 2019; Mozaffari Nejad et al., 2019; De Roma et al., 2017; Mahmoudi and Norian, 2015; Bahrami et al., 2016; Heshmati and Milani, 2010; Kamkar, 2005). In the present study, although the estimated milk contaminations in winter $\left(61.25 \pm 28.91 \mathrm{ngkg}^{-1}\right)$ and summer $\left(54.20 \pm 25.51 \mathrm{ngkg}^{-1}\right)$ were not statistically different $(P=0.279)$, the contaminations above $81 \mathrm{ngkg}^{-1}$ or the median $\left(70 \mathrm{ngkg}^{-1}\right)$ were mostly detected in winter and the chance of contaminations above median was 5.33 times in winter. Interestingly, the $\mathrm{AFB}_{1}$ contamination of feeds, as the main source of contamination, was significantly higher in summer than in winter ( $\left.\mathrm{P}<0.002\right)$. As the main source of milk contamination, the $\mathrm{AFB}_{1}$ contaminations of feeds have been reported to be higher in winter (Bahrami et al., 2016; Dimitrieska-

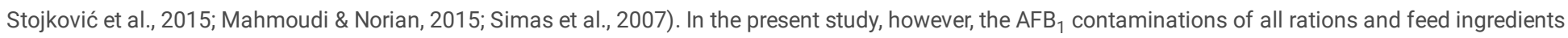
were significantly higher in summer than in winter (see below).

Aflatoxin $\mathrm{B}_{1}$ in Feed ingredients and TMRs 
Ninety percent of the examined ingredients $(n=207 / 230)$ were positive for $A F B_{1}, 10 \%(n=23 / 230)$ had non-detectable values and $13.9 \%(n=32 / 230)$ had values above $101.25 \mathrm{ngkg}^{-1}$. All ingredients exhibited higher contaminations in summer $(P<0.05)$. The non-detectable $A_{F} B_{1}$ values were mostly seen in winter $(n=17 / 23)$ and the values above $101.25 \mathrm{ngkg}^{-1}$ were detected mainly in summer $(n=27 / 32)$. These differences could be due to the synergistic effects of ambient temperature and feed moisture on behavior of mycotoxigenic fungi in summer, which is affected by climatic changes at any stage of production chain (Paterson and Lima, 2010; Magan et al., 2011; Guchi, 2015; Alvarado et al., 2017).

Iranian studies focusing on $\mathrm{AFB}_{1}$ contamination of dairy feeds are not abundant. Bahrami et al. (2016) examined 160 samples of corn silage, alfalfa hay, straw and barley $\left(n=40\right.$ each), detected $A_{F B}$ in $82.5 \%$ of samples with levels above $5,000 \mathrm{ngkg}^{-1}$ (Iranian standard) in $65 \%$ of corn silage and $10 \%$ of straw samples. Different prevalence rates have been reported for aflatoxin contamination of feeds from various countries such as China (42\%; Zheng et al., 2013), Costa Rica (33\%; Chavarría et al., 2015), Portugal (22\%; Abrunhosa et al., 2016), Tanzania (65\%, Mohammed et al., 2016) and Turkey (61.7\%, Kocasari et al., 2013; 34.9\% Sahin et al., 2013; 26.3\%, Sahin et al., 2016). A global study covering 2009 to 2011 (Rodrigues and Naehrer, 2012) and 4,627 feed samples reported aflatoxin contamination in $33 \%$ of samples (mean: $21,000 \mathrm{ngkg}^{-1}$ ). A survey of world reports on 1,113 feed samples from 44 countries between 2012 and 2015 (Kovalsky et al., 2016) reported that only a few samples from Africa and Europe exhibited levels exceeding 20,000 ngkg ${ }^{-1}$, which is the maximum permitted level for some non-dairy animals (Kunsagi et al., 2011; ISIRI, 2016). Different prevalence rates among various studies might be due to climatic variations and different detection methods. Ghali et al. $(2008,2009)$ detected aflatoxins in $76.4 \%$ and $62 \%$ of sorghum samples with ELISA and HPLC methods, respectively.

Consistent with the feed ingredients, the TMRs also had higher contaminations in summer. The total daily AFB 1 contents of bunk TMRs were $537.05 \pm 558.79$ $\mathrm{ng}$ in winter and $1375.50 \pm 905.02 \mathrm{ng}$ in summer. It is implied from some evidences that these levels were low enough to result in milk $\mathrm{AFM}_{1}$ levels below 50 $\mathrm{ngkg}^{-1}$. About 0.3 to $6.2 \%$ of $\mathrm{AFB}_{1}$ is secreted as $\mathrm{AFM}_{1}$ to milk (Becker-Algeri et al., 2016; FAO/WHO, 2002) and in cows producing $>30 \mathrm{~kg}$ milk/day, about $6 \%$ of $\mathrm{AFB}_{1}$ may be transformed to $\mathrm{AFM}_{1}$ (Britzi et al., 2013). In the present study, with milk production of $30.5 \mathrm{~kg} / \mathrm{cow} / \mathrm{day}$ and assuming $6 \%$ bio-transformation, the $\mathrm{AFM}_{1}$ content of milk could range from $1.05-2.7 \mathrm{ngkg}^{-1}$ (totally 32 to $82.5 \mathrm{ng}$ ). However, the estimated $\mathrm{AFM}_{1}$ levels were $61.25 \pm 28.91 \mathrm{ngkg}{ }^{-1}$ in winter and $54.20 \pm 25.51 \mathrm{ngkg}^{-1}$ in summer. Thus, the true contaminations of the ingredients could be higher than the detected levels. As the AFB 1 content of TMR samples taken from bunks were higher than those calculated from the contaminations of separate ingredients, the contamination could be increased during processing. Common devices such as mills and mixing wagons (Pinotti et al., 2016) and remainders of contaminated feeds in wagons, preparation areas and bunks may increase the level of aflatoxin in the ration. The difference could also be due to the small quantities of samples taken from huge volumes of feeds. The lack of correlation between feed $\mathrm{AFB}_{1}$ and milk $\mathrm{AFM}_{1}$ could be due to variations in daily intake of $\mathrm{AFB}_{1}$.

Seasonal variations in $\mathrm{AFB}_{1}$ in feeds and $\mathrm{AFM}_{1}$ in milk

With elevated dietary $\mathrm{AFB}_{1}$ levels and high chance of TMR contaminations above median $(716 \mathrm{ng} / \mathrm{kg} ; \mathrm{OD}=5.57 ; \mathrm{P}=0.002)$ in summer, higher levels of $A F \mathrm{M}_{1}$ in milk would be expected, but the findings were opposite. The reason could be the affection of liver functions by heat stress in summer (Bernabucci et al., 2010;

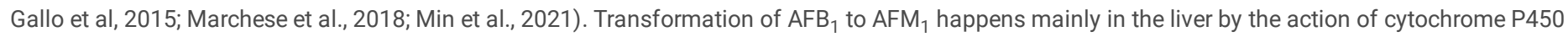
enzymes (Marchese et al., 2018; Min et al., 2021), involved in a vast variety of reactions. Compromised liver functions and reduced hepatic enzyme activities have been observed in heat stressed cows (Bernabucci et al., 2010; Fan et al., 2018). Compromised liver activities may be long lasting as it was observed in mid lactation cows (Mohebbi-Fani et al., 2020). Lower dry matter intakes in summer could also have a role in this finding. So, non-dietary factors affecting the metabolism of $A_{F B}$ may fade the effect of higher intakes of toxin in summer. Paradoxically, if the general health of the cows is improved, the $A F M_{1}$ contamination of milk would potentially be elevated. Thus, the most logic way to control the aflatoxin contamination in milk is to control the critical contaminants of the rations.

Critical feed contaminants

The $\mathrm{AFB}_{1}$ content of all ration ingredients showed some correlations with $\mathrm{AFB}_{1}$ content of TMRs. The strongest correlations were seen for grain mix and corn silage, which had the highest proportions in the rations. However, the impact of all other ingredients should also be taken into account. Beet pulp also could be an important source of contamination (strong correlations) regardless of its low incorporation or absence in rations. Alfalfa hay also could be an important contaminant, despite that its $\mathrm{AFB}_{1}$ contents were not correlated with those of the total rations. It is incorporated in nearly all rations in considerable amounts in Iranian farms and may be substituted for corn silage in many conditions. The co-occurrence and the synergistic effects of various mycotoxins even at relatively low levels (Kovalskey et al., 2016) should be kept in mind. In addition to controlling the $\mathrm{AFB}_{1}$ contaminations of feed ingredients, tainting of feedstuffs during processing should also be controlled. Intensifying the controlling measures in summer, with probable higher contaminations of feedstuffs, may reduce the overall milk contamination. Responses to these efforts may be rapid as $A_{F} M_{1}$ enters the milk $12-24$ hours after consumption of $A F B_{1}$ and drops to non-detectable levels about 72 hours of removal of $\mathrm{AFB}_{1}$ from ration (Pettersson, 1998). Based on the results of this study, a great majority of milk produced in the studied farms could have $\mathrm{AFM}_{1}$ contaminations below the ISIRI limit $\left(100 \mathrm{ngkg}^{-1}\right)$. Contaminations below 50 ngkg ${ }^{-1}$ appear to be achievable and affordable in the studied farms.

\section{Declarations}

\section{Acknowledgement}

The authors would like thank the industrial dairy farms in Qazvin province that participated in this study. The technical cooperation of central diagnostic laboratory of the Veterinary Organization, Qazvin, Iran is highly appreciated. 
Funding: this study was financially supported by Shiraz University (Grant number: 97GCU2M235008)

Conflicts of interest/Competing interests: the authors do not have any conflict of interest.

Ethics approval: not applicable.

Consent to participate: the studied farms were willing for cooperation and provision of the requested data.

Consent for publication: the authors are aware of this manuscript and the actions for its publication.

Availability of data and material: the data are available on request.

Code availability: not applicable

Authors' contributions

literature review, sampling, data collection and measurements were done by Ravel Gholampour-Aghdami. Designing and managing the work were performed by Mehdi Mohebbi Fani and Arash Omidi who also prepared the first draft of the manuscript. Aria Rasooli and Maryam Ansari-Lari advised on raw data and corrected the text. Statistical analysis was performed by Maryam Ansari-Lari.

\section{References}

1. Alvarado, AM; Zamora-Sanabria, R and Granados-Chinchilla, F (2017). A focus on aflatoxins in feedstuffs: Levels of contamination, prevalence, control strategies, and impacts on animal health. In: Abdulra'Uf, L., editor. Aflatoxin-Control, Analysis, Detection and Health Risks. Intech, London, pp116-152.

2. Abrunhosa L, Morales H, Soares C, Calado T, Vila-Chã AS, Pereira M and Venâncio A (2016). A review of mycotoxins in food and feed products in Portugal and estimation of probable daily intakes. Critical Reviews in Food Science and Nutrition. 52:249-265

3. Bahrami R; Shabdizi Y and Nikousefat Z (2016). Occurrence and seasonal variation of aflatoxin in dairy cow feed with estimation of aflatoxin M1 in milk from Iran. Food Agr Immunology. 27:388-400.

4. Beheshti HR and Asadi M (2014). Aflatoxins in animal feed in Iran. Food Additives and Contaminants. Part B Surveillence. 7:40-42. doi: $10.1080 / 19393210.2013 .834485$

5. Becker-Algeri, T; Castagnaro, D; Camila de Souza KB; Drunkler, DA and Badiale-Furlong, E (2016). Mycotoxins in Bovine Milk and Dairy Products. Rev J Food Sci., 81: Nr. 3, 2016.

6. Bernabucci, U; Lacetera, N; Baumgard, LH; Rhoads, RP; Ronchi, B and Nardone, A (2010). Metabolic and hormonal acclimation to heat stress in domesticated ruminants. Animal, 4:116-1183.

7. Britzi, M; Friedman, S; Miron, J; Solomon, R and Cuneah, O (2013). Carry-Over of Aflatoxin B1 to Aflatoxin M1 in High Yielding Israeli Cows in Mid- and Late-Lactation. Toxins, 5: 173-183.

8. Chavarría G, Granados-Chinchilla F, Alfaro-Cascante M and Molina A (2015). Detection of aflatoxin M1 in milk, cheese and sour cream samples from Costa Rica using enzyme assisted extraction and HPLC. Food Additives and Contaminants Part B Surveillance. 8:128-135. DOI:

$10.1080 / 19393210.2015 .1015176$

9. Commission, E. Commission Regulation (EU) No 574/2011 of 16 June 2011 amending Annex I to Directive 2002/32/EC of the European Parliament and of the Council as regards maximum levels for nitrite, melamine, Ambrosia spp. and carry-over of certain coccidiostats and histomonostats and consolidating Annexes I and II thereto. Off. J. Eur. Union 2011, L159, pp7-24.

10. De Roma, A; Rossini, C; Ritieni A; Gallo, P and Esposito, M (2017). A survey on the Aflatoxin M1 occurrence and seasonal variation in buffalo and cow milk from Southern Italy. Food Control, 81:30-33.

11. Dimitrieska-Stojković, E; Stojanovska-Dimzoska, B; llievska, G; Uzunov, R; Stojković, G; Hajrulai-Musliu, Z and Jankuloski, D (2015). Assessment of aflatoxin contamination in raw milk and feed in Macedonia during 2013. Food Control, 59:201-206.

12. Fan, C; Su, D; Tian, H; Li, X; Li, Y; Ran, L; Hu, R and Cheng, J (2018). Liver metabolic perturbations of heat-stressed lactating dairy cows. Asian-Aust. J. Anim. Sci., 31:1244-1251.

13. FAO/WHO (2002). Evaluation of certain mycotoxins in food: fifty-sixth report of the Joint FAO/WHO Expert Committee on Food Additives. https://apps.who.int/iris/handle/10665/42448. Accessed at 2021.8.21.

14. FAO/WHO (2001). Comments on the Draft Maximum Level for Aflatoxin M1 in Milk (Agenda Item 15a), Report of the 33rd Session of the Codex Committee on Food Additives and Contaminants. http://www.fao.org/3/Y0474E/y0474e00.htm\#Contents.Accessed at 2021.8.21.

15. Gallo, A; Giuberti G; Frisvad, JC; Bertuzzi, T and Nielsen KF (2015). Review on mycotoxin issues in ruminants: Occurrence in forages, effects of mycotoxin ingestion on health status and animal performance and practical strategies to counteract their negative effects. Toxins, 7:3057-3111.

16. Ghali R, Hmaissia-khlifa K, Ghorbel H, Maaroufi K and Hedili A (2008). Incidence of aflatoxins, ochratoxin A and zearalenone in tunisian foods. Food Control. 19:921-924. DOl:10.1016/j.foodcont.2007.09.003

17. Ghali R, Belouaer I, Hdiri S, Ghorbel H, Maaroufi K and Hedilli A (2009). Simultaneous HPLC determination of aflatoxins B1, B2, G1 and G2 in Tunisian sorghum and pistachios. Journal of Food Composition and Analysis. 22:751-755. DOI: 10.1016/j.jfca.2009.04.009

18. Guchi, E (2015). Implication of aflatoxin contamination in agricultural products. Am. J. Food Nutr., 3:12-20. 
19. Hamzeh Pour, S; Mahmoudi, S; Masoumi, S; Rezaie, S; Barac, A; Ranjbaran, M; Oliya, S; Mehravar, F; Sasani, E; Noorbakhsh, F and Khodavaisy, S (2020). Aflatoxin M1 contamination level in Iranian milk and dairy products: a systematic review and meta-analysis. World Mycotoxin J. 13: 67-82

20. Heshmati, A and Milani JM (2010). Contamination of UHT milk by aflatoxin M1 in Iran. Food Control, 21:19-22.

21. Institute of Standards and Industrial Research of Iran (ISIRI) (2020). Food and feed- Maximum tolerated level of mycotoxins. National Standard No. 5925. 1st revision. http://www.isiri.org/portal/files/std/164.doc. Accessed 2 July 2021.

22. Institute of Standards and Industrial Research of Iran (ISIRI) (2016). Milk and milk products-raw milk-specifications and test methods. National Standard No. 164. 3rd revision. http://www.isiri.org/portal/files/std/164.doc. Accessed 2 July 2021.

23. Iranian Veterinary Organization (IVO) (2008). Inspection and sanitary control of raw milk. Procedure No 84.44.05. www.ivo.ir., accessed at 2021.08.01

24. Iranian Veterinary Organization (IVO) (2005). Sampling of primary ingredients and prepared feeds. Procedure No 84.44.06. www.ivo.ir., accessed at 2021.08.01

25. Kamkar, A (2005). A study on the occurrence of aflatoxin M1 in raw milk produced in Sarab city of Iran. Food Control, 16:593-599.

26. Khaneghahi Abyaneh, H; Bahonar, A; Noori, N; Yazdanpanah, H and Shojaee Ali Abadi, MH (2020). The overall and variations of Aflatoxin M1 contamination of milk in Iran: A systematic review and meta-analysis study. Food Chem., 310:125848. doi: 10.1016/j.foodchem.2019.125848.

27. Kocasari, FS; Mor, F; Oguz, MN and Oguz, FK (2013). Occurrence of mycotoxins in feed samples in Burdur Province, Turkey. Env. Mon. Assess., 185:49434949.

28. Kovalsky P, Kos G, Nährer K, Schwab C and Jenkins T (2016). Co-occurrence of regulated, masked and emerging mycotoxins and secondary metabolites in finished Feed and Maize-An Extensive Survey. Toxins. 8:1-29. DOI: 10.3390/toxins8120363

29. Kunsagi, Z; Breidbach, A and Stroka, J (2012). Report on the 2011 Proficiency Test of the European Union Reference Laboratory for Mycotoxins, for the Network of National Reference Laboratories. Determimation of Aflatoxin B1 in Baby food, Maize powder, Animal Feed and Test solution. European Commission, Joint Research Centre. European Union, 2012. https://publications.jrc.ec.europa.eu/repository/handle/JRC68705

30. Magan, N.; Medina, A. and Aldred, D (2011). Possible climate-change effects on mycotoxin contamination of food crops pre- and postharvest. Plant Pathol. 60:150-163.

31. Mahmoudi, R and Norian, R (2015). Aflatoxin B1 and M1 contamination in cow feeds and milk from Iran. J. Food Agri. Immun., 26:131-137.

32. Marchese, S; Polo, A; Ariano, A; Velotto, S; Costantini, S and Severino, L (2018). Aflatoxin B1 and M1: Biological Properties and Their Involvement in Cancer Development. Toxins, 10:214, doi:10.3390/toxins10060214

33. Mashak, Z; Jafari Sohi, H; Heshmati, A and Mozaffari Nejad, AS (2016). Assessment of Aflatoxin M1 Contamination in UHT Flavored Milk Samples in Karaj, Iran. Iranian J. Pharmaceutical Res., 15: 407-411.

34. Min, L; Fink-Gremmels, J; Li, D; Tong, X; Tang, J; Nan, X; Yu, Z; Chen, W and Wang, G (2021). An overview of aflatoxin B1 biotransformation and aflatoxin M1 secretion in lactating dairy cows. Anim. Nutr., 7:42-48.

35. Mohammed S, Munissi JJ and Nyandoro SS (2016). Aflatoxin M1 in raw milk and aflatoxin B1 in feed from household cows in Singida, Tanzania. Food Additives and Contaminants Part B Surveillance. 9:85-90. DOI: 10.1080/19393210.2015.1137361

36. Mohebbi-Fani, M; Omidi, A; Mirzaei, A; Nazifi, S; Pour-Tajabadi, E and Badkubeh-Hazaveh, M (2020). Compromised liver functions during the breeding period of clinically healthy Holstein cows. Iranian J Vet. Res., 20:89-95.

37. Mozaffari Nejad, AS; Heshmati, A and Ghiasvand,T (2019). The Occurrence and Risk Assessment of Exposure to Aflatoxin M1 in Ultra-High Temperature and Pasteurized Milk in Hamadan Province of Iran. Osong Publ. Health Res. Perspective, 10:228-233.

38. Rezaei M, Mohammadpourfard I, Malekirad AA, Ghasemikhah R and Sayadi M (2014). Aflatoxin Contamination of Feed Materials in Qom Province, Iran. Iranian Journal of Toxicology. 8:1025-1027.

39. Robinson, PH and Meyer D (2010). Total Mixed Ration (TMR) Sampling Protocol. University of California, Publication No. 8413. ANR Publication. . Accessed at 2021.8.21.

40. Rodrigues I and Naehrer K (2012). A three-year survey on the worldwide occurrence of mycotoxins in feedstuffs and feed. Toxins. 4:663-675. D0I: 10.3390/toxins4090663

41. Paterson RM and Lima N (2010). How will climate change affect mycotoxins in food? Food Research International. 43:1902-1914. DOI: 10.1016/j.foodres.2009.07.010

42. Pettersson, H. (1998). Carryover of aflatoxin from feeding stuff to milk. Report of Department of Animal

43. Nutrition and Management. Swedish University of Agricultural Sciences, Upsala, Sweden. p. 21.

44. Pinotti L, Ottoboni M, Giromini C, Dell'Orto V and Cheli F (2016). Mycotoxin contamination in the EU feed supply chain: A focus on cereal byproducts. Toxins. 8:45. DOI: 10.3390/toxins8020045

45. Sahin, HZ; Celik, M; Kotay, S and Kabak, B (2016). Aflatoxins in dairy cow feed, raw milk and milk products from Turkey. Food Add. Contaminants, Part B Surveillance. 9:152-158.

46. Sahin, T; Aksakal, DH; Sünnetci, S; Keser, O and Eseceli H (2013). Detection of aflatoxin, zearalenone and deoxynivalenol in some feed and feedstuffs in Turkey. Pakistan Vet. J., 34:459-463

47. Simas, MMS; Botura, MB; Correa, B; Sabino, M; Mallmann, CA; Bitencourt, TCBSC and Batatinha, MJM (2007). Determination of fungal microbiota and mycotoxins in brewers grain used in dairy cattle feeding in the State of Bahia, Brazil. Food Control, 18:404-408.

48. Škrbić, B; Živančev, J; Antić, I and Godula, M (2013). Levels of aflatoxin M1 in different types of milk collected in Serbia: Assessment of human and animal exposure. Food Control, 40:113-119. 
49. Sulzberger, SA; Melnichenko, S and Cardoso, FC (2017). Levels of aflatoxin M1 in different types of milk collected in Serbia: Assessment of human and animal exposure. J. Dairy Sci., 100:1856-1869.

50. Tajik, H; Moradi, M; Razavi Rohani, SM and Hadian M (2016). Determination of Aflatoxin M1 in Pasteurized and UHT Milk in West-Azerbaijan Province of Iran. J. Food Qual. Haz. Cont., 3:37-40.

51. Tsakiris, IN; Tzatzarakis, MN; Alegakis, AK; Vlachou, MI; Renieri, EA and Tsatsakis, AM (2013). Risk assessment scenarios of children's exposure to aflatoxin M1 residues in different milk types from the Greek market. Food Chem. Toxicol., 56:261-265.

52. Zheng, N; Wang, JQ; Han, RW; Zhen, YP; Xu, XM and Sun, P (2013). Survey of aflatoxin M1 in raw milk in the five provinces of China. Food Add. Contaminants, Part B Surveillance. 6:110-115.

\section{Figures}

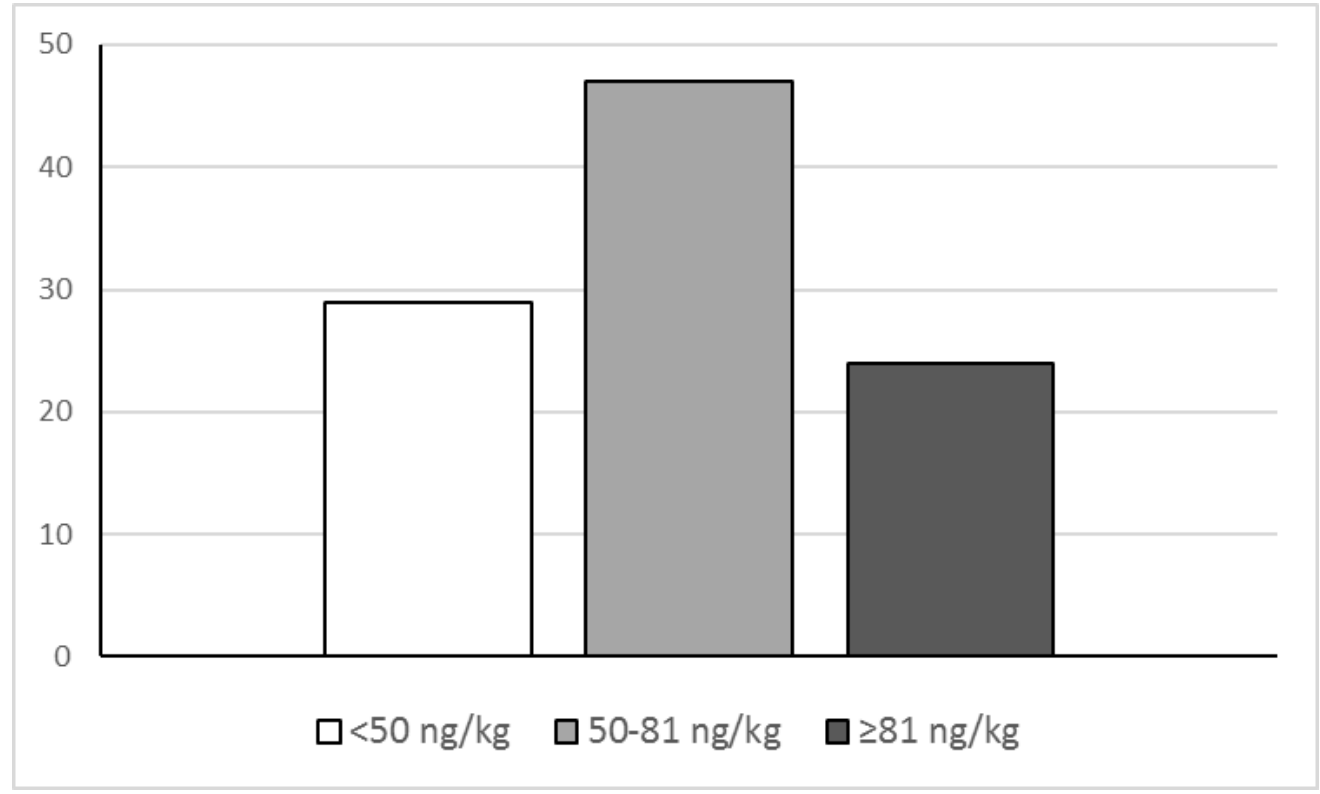

Figure 1

Frequencies (\%) of bulk milk samples ( $\mathrm{n}=72$ ) with AFB1 contaminations below 50, 50-81 and above $81 \mathrm{ngkg}-1$.

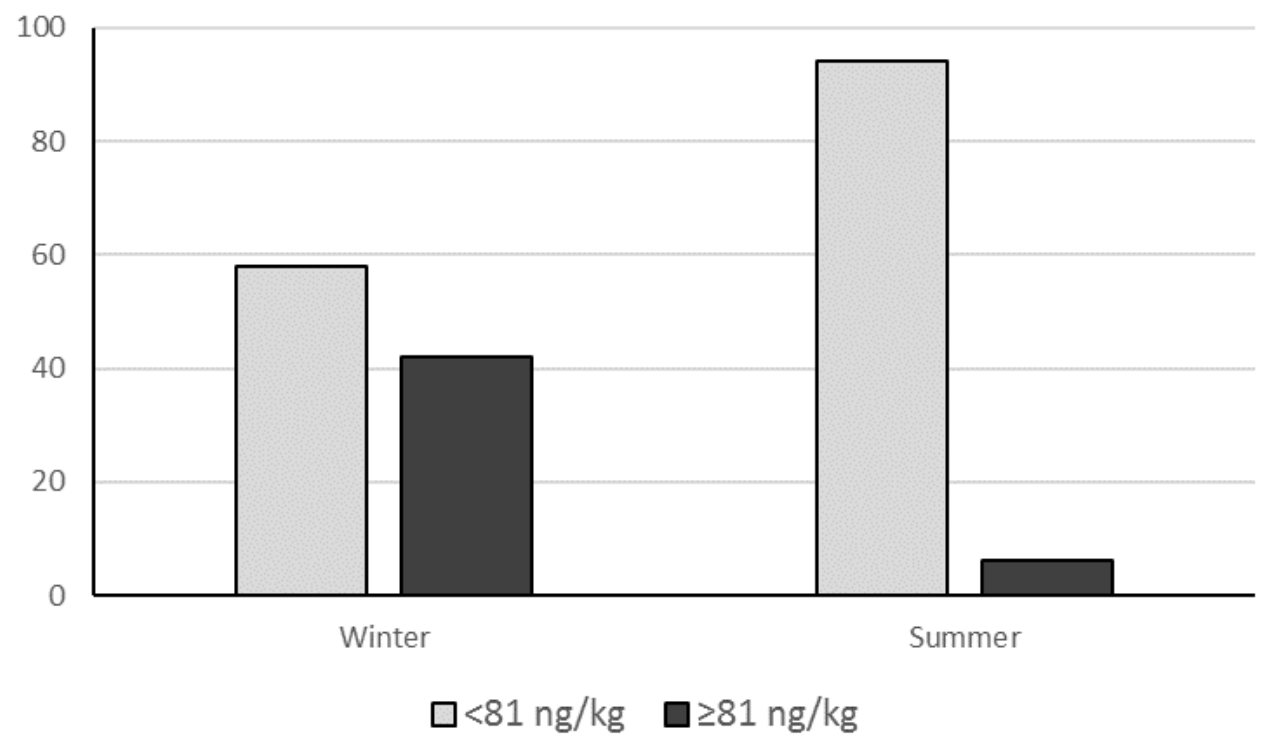

Figure 2

Frequencies (\%) of bulk milk samples with AFM1 contaminations below and above $81 \mathrm{ng} / \mathrm{kg}$ during winter and summer ( $\mathrm{n}=36$ each season). 


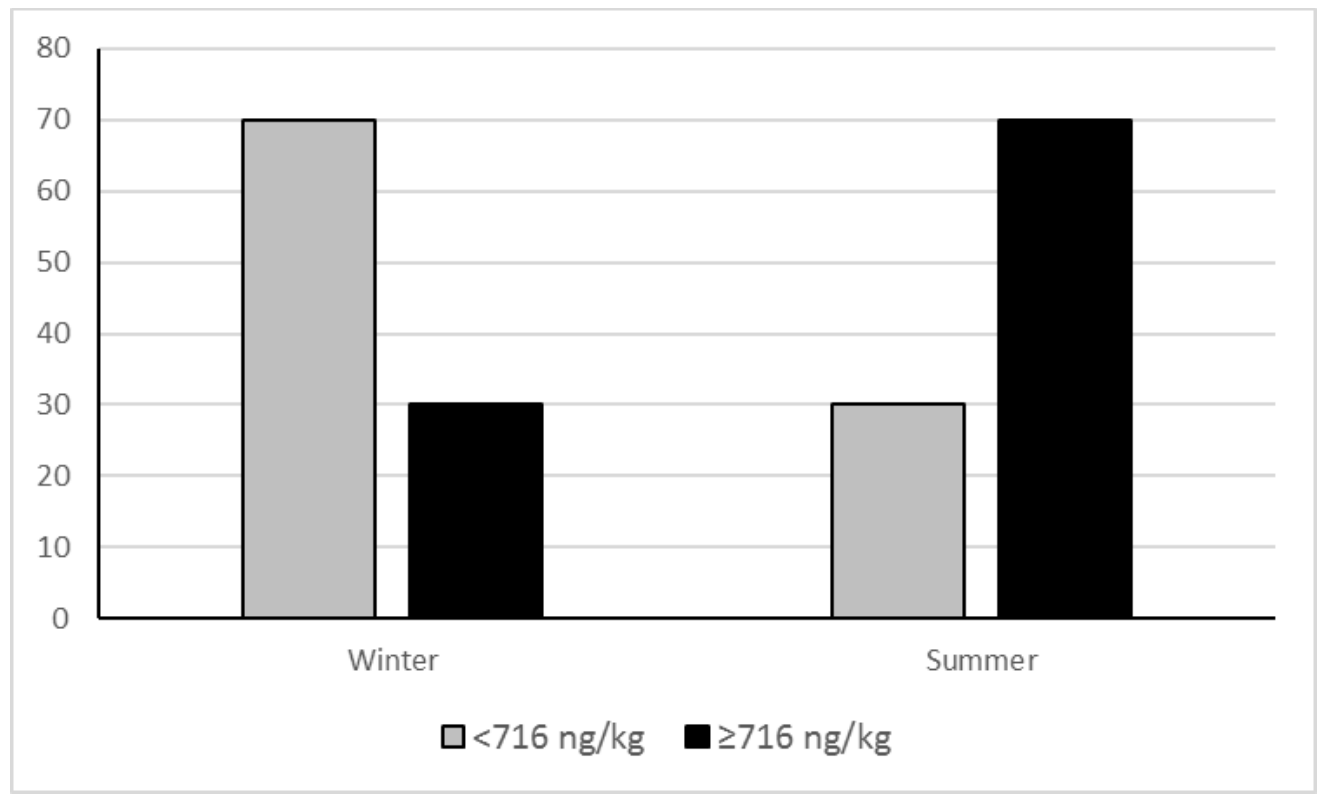

Figure 3

Frequencies (\%) of bunk TMR samples with AFB1 contaminations below and above median (716 ngkg-1) during winter and summer ( $\mathrm{n}=24$ each season). 